\title{
High Speed Electro-absorption Modulator for Long Range Retroreflective Free Space Optics
}

\author{
C. Quintana, Q. Wang, D. Jakonis, X. Piao, G. Erry, D. Platt, Y. Thueux, A. Gomez, G. Faulkner, H. \\ Chun, M. Salter, and D. O'Brien Member, IEEE
}

\begin{abstract}
In this work we present the design and implementation of a pixelated electro-absorption modulator (EAM) based modulating retroreflector (MRR) for high speed optical wireless communications. The modulator is based on a multiple quantum well (MQW) structure embedded in an asymmetric Fabry-Perot (FP) cavity. This MRR was used in an outdoor link, operating at $150 \mathrm{Mbps}$ with a bit error rate (BER) of $1.22 \times 10^{-6}$ at a range of $200 \mathrm{~m}$. The system was also tested in laboratory controlled conditions achieving a data rate of $200 \mathrm{Mbps}$ with a BER of $2 \times 10^{-4}$. To the best of our knowledge, this is the fastest retroreflective free space optics (RFSO) demonstration in both indoor and outdoor environments.
\end{abstract}

Index Terms - Free-space optical communications, Quantum well devices, modulating retroreflector

\section{INTRODUCTION}

$\mathrm{R}^{\mathrm{s}}$ ecent market studies forecast substantial growth in the use of nano/microsatellites for a wide range of scientific, commercial and military applications [1]. This trend is led by CubeSat platforms, as they represent a low-cost means of performing Earth Observation in low-Earth-orbit (LEO) [2]. These satellites are heavily constrained by cost, size, and power consumption, with a total mass typically below $5 \mathrm{~kg}$ [3]. Thus, an efficient, compact high data-rate communications module is required, making the downlink (satellite to ground station) implementation very challenging.

Currently, CubeSat downlinks rely on radio frequency (RF) communications. These systems are reliable, but limited to tens of kbps in data rate to the transmitted power constraints and high beam divergence and consequent losses [4]. Free space optics (FSO) systems can provide narrow optical beams with very high directivity. This allows for significantly higher data rates with increased transmission security [5-6]. Furthermore, FSO systems make use of unregulated band avoiding the saturation of RF spectrum [7].

Within FSO, retroreflective free space optic (RFSO) architectures are well-suited to CubeSat applications as the localization and tracking complexities are potentially moved to the ground station. In RFSO communications, a high power continuous wave $(\mathrm{CW})$ laser beam is launched from a base

Manuscript received December 15, 2016; revised x; accepted x. Date of publication $x$

This work was supported by the European Commission. H2020 Grant Agreement: 637595.

C. Quintana A. Gomez, G. Faulkner, H. Chun and D. O'Brien are with the Department of Engineering Science, University of Oxford, Oxford OX1 3PJ, U.K. (email: crisanto.quintanasanchez@eng.ox.ac.uk) station (BS) towards a modulating retroreflector (MRR) located at distant terminal. This interrogating laser beam can be modulated at the BS to establish an uplink [8]. The downlink is automatically aligned as the MRR modulates the interrogating beam and retro-reflects it back to the ground station in the same direction.

Although an attractive concept, the practical feasibility of such a link is not proven, and an EC funded project (C3PO) is currently undertaking a feasibility study. One focus of this program is the implementation of compact and high bandwidth MRRs [9]. MRR modulation can be achieved using liquid crystal (LC), micro-electro-mechanical systems (MEMs), and electro-absorption modulator (EAMs) optical shutters. LC and MEMs device data rates are currently limited to a few kbps and Mbps respectively $[8,10]$. However, data rates of $70 \mathrm{Mbps}$ in lab conditions and $45 \mathrm{Mbps}$ in outdoor applications, have been achieved using multiple quantum wells (MQW) based EAMs [11-12].

In this work, we present the design and implementation of a novel MRR based on an array of MQW based modulators. This device is used in a data link operating at $150 \mathrm{Mbps}$ at a range of $200 \mathrm{~m}$ in an outdoor environment with atmospheric turbulence. Data rates of $200 \mathrm{Mbps}$ obtained in lab conditions are also reported. To the best of our knowledge, these are the fastest demonstrations of RFSO links in both indoors and outdoors scenarios.

The paper is organized as follows. In Section II, we present the MRR system design including MQW EAM, driver electronics and optics. Section 3 describes the experimental results and discussion. Finally, conclusions are provided in section 4 .

\section{MRR DESIGN}

The link budget of an RFSO system has been shown to be proportional to the $\mathrm{D}_{\mathrm{MRR}}{ }^{4}$, where $\mathrm{D}_{\mathrm{MRR}}$ is the optical aperture of the retroreflector [13]. This dependence suggests the use of large MRR apertures to increase the link range. However, the switching rate of MQW-based modulators is inversely proportional to their active area. A larger modulator area also increases the electrical power consumption of the device [13].

In order to increase the MRR aperture whilst keeping the

Q. Wang, D. Jakonis, X. Piao, D. Platt and M. Salter are with Acreo Swedish ICT AB, Electrum 236, 16440, Kistra, Sweden. (email: Qin.Wang@ acreo.se)

G. Erry and Y. Thueux are with Airbus Group Innovations, Quadrant House, Celtic Springs, Coedkernew, Newport, NP10 8FZ, UK 
MQW's active area constant, a cat's eye retroreflector architecture is adopted here. The design is based on an optical system that focuses the interrogating beam into the EAM, working in reflective mode. Figure 1 shows the optical system and modulator arrangement. Some electronics is also required to drive the EAM with suitable reverse voltage levels. These three subsystems are described in detail in this section.

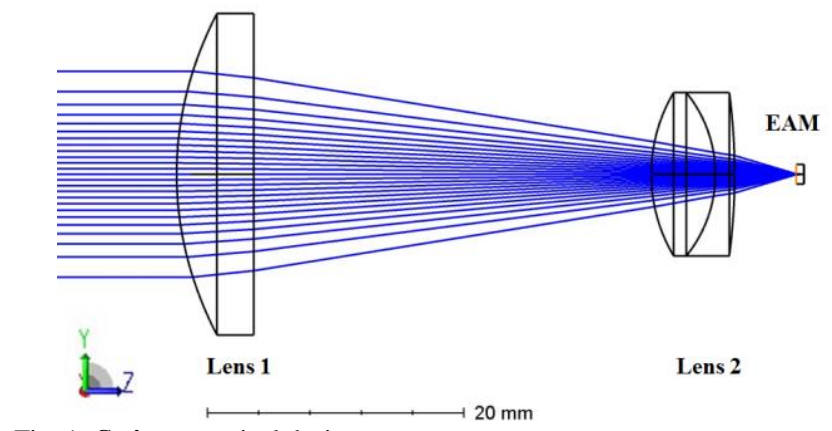

Fig. 1. Cat's eye optical design.

For this particular application, a MRR diameter of $20 \mathrm{~mm}$ and a field of view (FOV) of $\pm 1.4^{\circ}$ are targeted. These specifications are sufficient for LEO CubeSat applications, where current commercial attitude determination and control systems provide pointing accuracies below $1^{\circ}$ [14]. This MRR will be interrogated using a $1550 \mathrm{~nm} \mathrm{CW}$ laser beam. This wavelength was selected because it allows the use of mature Cband technology and relaxes eye-safety constraints. A downlink data rate of Gbps is desired.

\section{1) EAM design}

EAMs are based on the quantum-confined Stark effect (QCSE), which describes the effect of an external electric field upon the light absorption spectrum of a quantum well. In this work, a surface-normal EAM optimised for C-band operation is designed. Surface-normal EAMs are polarisation insensitive and allow large format 2D array manufacturing. Nevertheless, they offer a limited Contrast Ratio (CR) because of the short interaction length between the incident light and the active medium. Typical measured CR are in the range of 2:1 [15]. This $\mathrm{CR}$ can be improved by using more quantum wells, albeit at the expense of increasing the insertion losses and the required bias voltage. The design presented here is based on 80 coupled InGaAs/InAlAs quantum wells embedded in an asymmetric Fabry-Perot (FP) cavity. This design leads to a $1.778 \mu \mathrm{m}$ thick modulator. This thickness has been shown to provide a good CR with an acceptable insertion loss [15].

In a generic cat's eye design, the diameter of the EAM $\left(D_{\text {EAM }}\right)$ is defined by:

$$
D_{E A M}=F \# D_{M R R} F F O V_{M R R}
$$

where $F \#, D_{M R R}$ and $F F O V_{M R R}$ represent the F number of the optical system, the MRR optical aperture and the full-angle field of view in radians, respectively. Low $F \#$ are desired to minimize the size of the modulator. However, values below 1.5 are difficult to achieve using commercial off-the-shelf (COTS) lenses. Considering that $\mathrm{F} \#$ limitation, a modulator diameter of $1.368 \mathrm{~mm}$ or higher is required to meet the FOV specification defined in this work.
A surface-normal EAM behaves as a 'parallel-plate' capacitance, whose value depends on the material dielectric constant, the area and thickness of the modulator. An InGaAs/InAlAs EAM with a $1.368 \mathrm{~mm}$ diameter would have an equivalent capacitance of $0.115 \mathrm{nF}$. This value, combined with a typical $50 \Omega$ driver impedance, will limit the EAM bandwidth to $27.5 \mathrm{MHz}$. Therefore, EAM pixelation is essential to meet the data rate requirements.

To achieve potential Gbps data rates, a pixelated $6 \times 6$ surfacenormal EAM was designed. Figure 2 shows the fabricated EAM mounted on a printed circuit board (PCB). This device was fabricated using standard semiconductor microfabrication technology at Acreo's ISO-9001 certificated clean-room facilities. The pixel size of the array is $250 \mu \mathrm{m} \times 250 \mu \mathrm{m}$ with a pitch of $280 \mu \mathrm{m} \times 280 \mu \mathrm{m}$, leading to an overall modulator array $1.65 \mathrm{~mm}$ square. Pixelation reduces the individual pixel capacitance to $3.9 \mathrm{pF}$, which corresponds to a modulator bandwidth of $811.7 \mathrm{MHz}$ (50 $\Omega$ driver). To drive each pixel individually a wire-bonding pad $(50 \mu \mathrm{m} \times 25 \mu \mathrm{m})$ is arranged on the top of the pixels, which blocks some of the incident optical beam. Thus, the fill factor of the EAM array is about $78.1 \%$ including the $30 \mu \mathrm{m}$ gaps between the pixels and the wire-bonding pad on the top of the pixels. This device operates in reflective mode by means of an aluminium mirror coated on the EAM's backside.

\section{2) Driver electronics}

The driver electronics module acts as an interface between the data source and the MRR, and drives each pixel with a suitable voltage level. Figure 2 shows the driver electronics block diagram. The driver consists of an LVDS to 3.3V TTL level translator and then a set of octal bus buffers implementing a tree distribution network to distribute a separate drive signal to each pixel. This ensures that each pixel is driven by its own bus buffer output stage.

The EAM reverse biasing is achieved by connecting the common cathode of the pixels to a fixed $3.8 \mathrm{~V}$ voltage power supply.

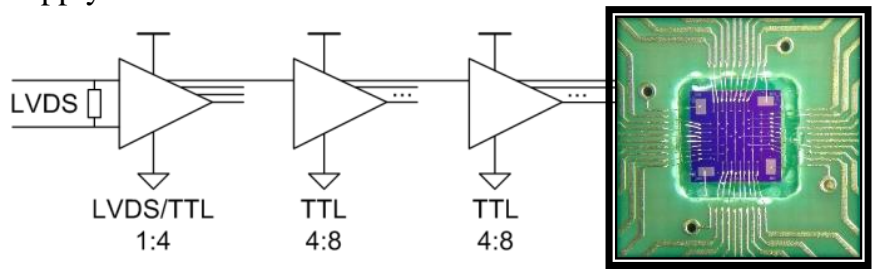

Fig. 2. Driver signal path for the 32 pixel EAM array. The 4 pixels on the corner are not connected to the driver.

\section{3) Optical system}

A two-lens optical system is used to meet the system specifications (aperture $=20 \mathrm{~mm}$ and $\mathrm{FOV}= \pm 1.4^{\circ}$ ). Figure 1 shows the optical layout. Lens 1 is an aspheric plano-convex (Thorlabs AL2550-C) with $25.4 \mathrm{~mm}$ aperture and $50 \mathrm{~mm}$ focal length. Lens 2 is an achromatic doublet (Thorlabs AC127-019C) with $12.5 \mathrm{~mm}$ aperture and a focal length of $19 \mathrm{~mm}$. The distance between them was set to $31 \mathrm{~mm}$ to minimize spherical aberrations. A precise z-location of the EAM on the focal plane of the optical system is essential to satisfy a diffraction-limited returned beam after reflection off the EAM. Additionally, the EAM must be placed normal to the optical axis in order to avoid 
any offset between the interrogator and the returned beam. Accurate mounting stages were used to achieve these requirements.

\section{RESULTS AND DISCUSSION}

In this section, we present the experimental tests performed on the EAM and the MRR above described.

\section{A. System characterization}

\section{1) EAM DC measurements}

To assess the performance of the fabricated EAM the absorption spectra was measured, using a Bruker V80 Fourier transform infrared spectroscopy (FTIR) spectrometer. Reverse bias voltages of $0,-3$ and $-6 \mathrm{~V}$ were used. Figure 3 shows the absorption spectra of the EAM at room-temperature $\left(\sim 23{ }^{\circ} \mathrm{C}\right)$. The CR peak, defined as the ratio between the absorption at $3 /-6 \mathrm{~V}$ and that at $0 \mathrm{~V}$, was obtained around $1540 \mathrm{~nm}$. At this wavelength, it can be also seen that a $3 \mathrm{~V}$ voltage swing provides a $\mathrm{CR}$ similar to that obtained at $6 \mathrm{~V}$. A double-pass (reflection mode) absorption of $11.5 \mathrm{~dB}$ and a CR of 1.25:1 were measured.

In MQW EAMs, the absorption spectra, and then the CR, shifts with temperature. Laboratory experiments showed a CR peak shift of $0.7 \mathrm{~nm} /{ }^{\circ} \mathrm{C}$ when the temperature was varied from 17 to $70{ }^{\circ} \mathrm{C}$. Therefore, a temperature control module is required to stabilize the EAM performance as a function of the wavelength, particularly for satellite applications where temperature fluctuates from -100 to $100^{\circ} \mathrm{C}$.

\section{2) MRR FOV measurements}

The FOV of the MRR was characterized in lab conditions. A monostatic interrogator is used to illuminate the MRR, which is mounted on a computer-controlled mechanical rotational stage $1 \mathrm{~m}$ from the interrogator. A $1552 \mathrm{~nm}$ laser beam illuminates the MRR, is returned and detected at the interrogator and converted into a voltage. Figure 4 shows the peak-to-peak normalized received voltage for different MRR angles of incidence (AoI). This shows a full-angle, half-power field of view of $2^{\circ}$. At $\pm 1.4^{\circ}$ AoI the MRR shows a reflected power drop of $30 \%$ with respect to the normal incidence. The figure also shows 5 drops on the modulated signal. This is caused by the $30 \mu \mathrm{m}$ gaps located between the EAM pixels.

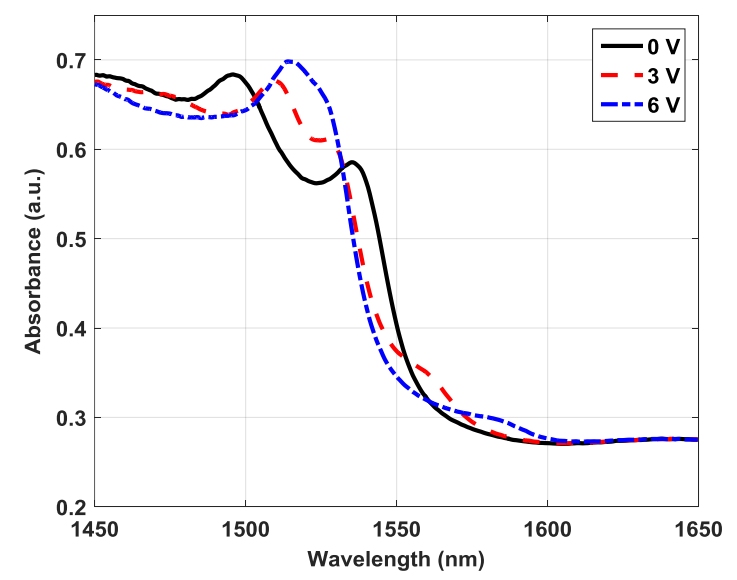

Fig. 3. EAM absorption spectra under different reverse bias voltages at roomtemperature $\left(23^{\circ} \mathrm{C}\right)$.

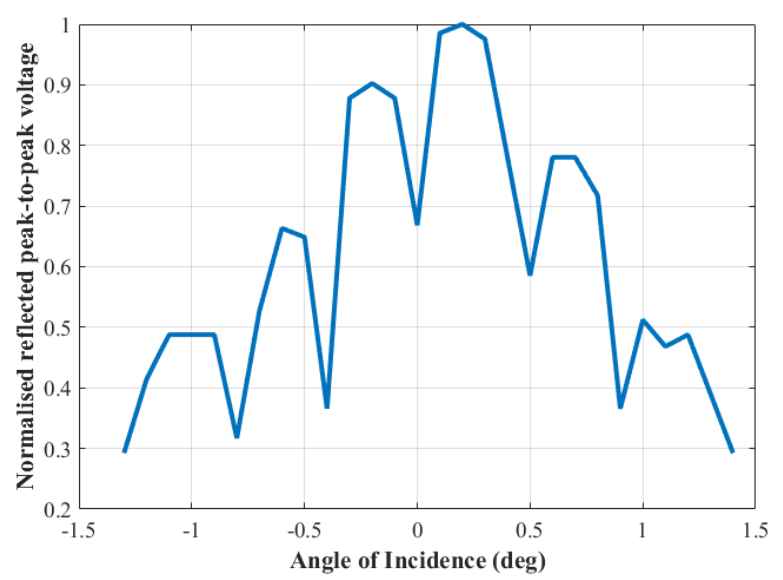

Fig. 4. MRR field of view characterization

\section{B. Data link demonstrations}

The MRR was tested in an outdoor environment affected by atmospheric turbulence. Figure 5 shows the experiment setup. The MRR was set up on tripod at a range of $200 \mathrm{~m}$ from a base station (BS) interrogator. A $15 \mathrm{dBm} 1549.5 \mathrm{~nm} \mathrm{CW}$ laser beam was used as the interrogating beam. This light source was collimated and expanded up to an $8 \mathrm{~cm}$ diameter beam at the output aperture of the BS. This beam was directed towards the MRR using a mechanical pan and tilt unit (PTU). Further detail of the BS architecture is provided in [16].

The MRR was modulated at $150 \mathrm{Mbps}$ non-return-to-zero (NRZ) on-off-keying (OOK), using a pseudo-random data stream (PRBS) (216-1) generated using a Xilinx ML507 board. The returned beam was detected with an InGaAs APD (Thorlabs APD110C) photo-detector. This device provides a 3 $\mathrm{dB}$ bandwidth of $50 \mathrm{MHz}$. The resulting electrical signal was finally captured using a high-bandwidth oscilloscope. Figure 6 shows both transmitted and detected signals. The received signal was post-processed using adaptive thresholding techniques to remove the low frequency intensity variation introduced by the scintillation. Over a period of $75 \mathrm{~s}$, corresponding to 4.915 Mbits, a bit error rate (BER) of $1.22 \mathrm{x}$ $10^{-6}$ was recorded. The test recording time was limited by the memory depth of the scope.

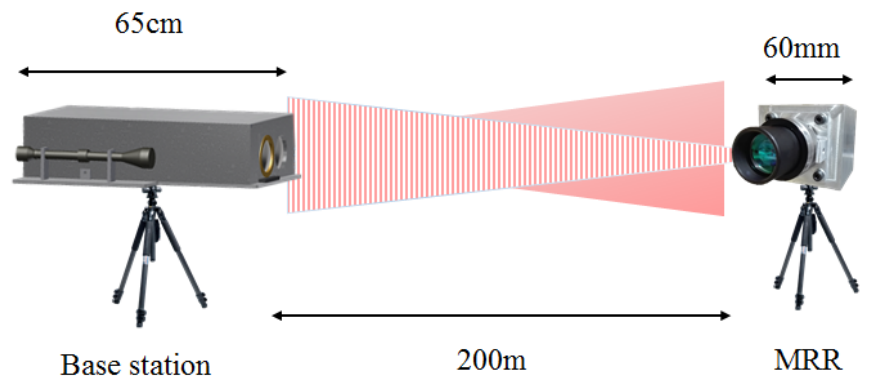

Fig. 5. Data link demonstration set-up. 


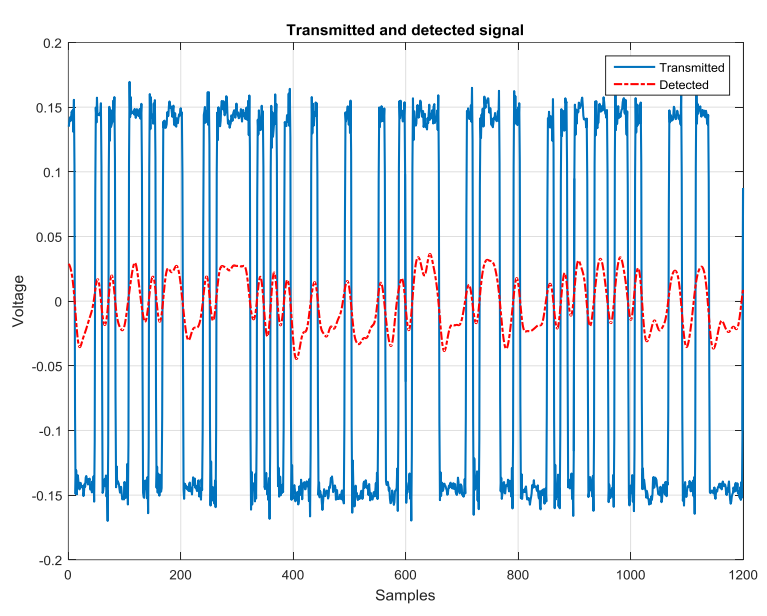

Fig. 6. Transmitted (blue) and post-processed (red) detected signals at 150 Mbps.

The MRR was also tested in lab conditions using the monostatic test bed described above. A maximum-likelihood sequence estimation (MLSE) was used to tackle both detector bandwidth limitation and MRR non-linear distortion, and a data-rate of $200 \mathrm{Mbps}$ was achieved at a BER of $2 \times 10^{-4}$. The equalizer uses a training sequence of 60 bits in a frame of 65535 bits. The overhead introduced by the equalizer is then less than $0.1 \%$, and the measured BER is sufficiently low that error free communications can be achieved by using a forward error correction (FEC) code [17]. The overhead introduced by these codes is $7 \%$. Although the estimated EAM bandwidth is approximately $800 \mathrm{MHz}$, the performance of the MRR is limited by the driver electronic and its integration with the EAM pixels. Non-linear distortions were also observed, caused by unmatched load driving. This limitation is expected to be solved in the future with a redesign of the driving electronics.

\section{CONCLUSIONS}

This paper reports the design and implementation of a high bandwidth MQW based MRR. An outdoor data link of 150 Mbps at a range of $200 \mathrm{~m}$ with a BER was $1.22 \times 10^{-6}$ was achieved. The system was also tested in lab conditions achieving a BER of $2 \times 10^{-4}$ at $200 \mathrm{Mbps}$. To the best of our knowledge, this is the fastest RFSO demonstration in both indoor and outdoor environments. An MRR FOV of $\pm 1.4^{\circ}$ was measured, which makes this device suitable for CubeSat LEO satellite applications.

OOK performance with limited $\mathrm{CR}$ modulators has been analysed in [18]. Future work should include the development of an analytical model to determine the optimum EAM thickness for this particular application. Additionally, an optimization of the driver/EAM integration is needed to improve the impedance mismatch and increase the date rate potentially up to $1 \mathrm{Gbps}$. An enhancement of the receiver sensitivity is also expected to increase the link range up to $1 \mathrm{~km}$.

\section{ACKNOWLEDGMENT}

Authors would like to thank I. Petermann, M. Karlsson, J. Lim, O. Öberg and B. Samel from Acreo Swedishs ICT and J. Brumfitt from the University of Oxford for their valuable support to this work.

\section{REFERENCES}

[1] B. Doncaster and J. Shulman, "2016 Nano/Microsatellite Market Forecast", Spaceworks Enterprices, Atlanta, Mar. 2016.

[2] J. Puig-Suari; C. Turner and W. Ahlgren, "Development of the standard CubeSat deployer and a CubeSat class PicoSatellite", Proc. IEEE Aerospace, 2001.

[3] J. Bouwmeester and J. Guo, "Survey of worldwide pico- and nanosatellite missions, distributions and subsystem technology", Acta Astronautica, vol. 67 (7-8), pp. 854-862, Oct. 2010.

[4] P. Muri and J. McNair, "A Survey of Communication Sub-systems for Intersatellite Linked Systems and CubeSat Missions", Journal of Communications, vol. 7 (4), pp. 258-264, 2012.

[5] D. M. Boroson, J. J. Scozzafava, D. V. Murphy, B. S. Robinson, M. I. T. Lincoln, "The Lunar Laser Communications Demonstration (LLCD), Space Mission Challenges for Information Technology", Third IEEE International Conference on SMC-IT, pp. 23-28, 2009.

[6] R. W. Kingsbury, D. O. Caplan and K. L. Cahoy, "Compact optical transmitters for CubeSat free-space optical communications", Proc. SPIE 9354, Free-Space Laser Communication and Atmospheric Propagation XXVII, 93540S, Mar., 2015.

[7] H. Kaushal and G. Kaddoum, "Optical Communication in Space: Challenges and Mitigation Techniques", IEEE Communications Surveys \& Tutorials, vol. PP (99), 2016.

[8] D.C. O'Brien, J.J. Liu and G.E. Faulkner, "Design and implementation of optical wireless communications with optically powered smart dust motes", IEEE Journal on Selected Areas in Communications, 27(9), p. 1646-1653, 2009.

[9] Y. Gouy, E. Steck, C. Quintana, G. Faulkner, et al., "The C3PO Project: A Laser Communication System Concept for Small Satellites", 4S Symposium, 1. Jun. 2016, Malta.

[10] W.S. Rabinovich, R. Mahon, M. Ferraro, J. Murphy, L. Mullen, B. Cohenour, J. Muth, and L. Ziph-Schatzberg, "Underwater Optical Modulating Retro-Reflector Links", Applications of Lasers for Sensing and Free Space Communications, San Diego, United States, 3 Feb. 2010

[11] W. S. Rabinovich, "Performance of cat's eye modulating retro-reflectors for free-space optical communications", Proc. SPIE, vol. 5550, pp. 104114, 2004.

[12] W.S. Rabinovich, P. G. Goetz, R. Mahon, L. Swingen, J. Murphy, M. Ferraro, H. Ray Burris, C. I. Moore, M. Suite, G. Charmaine Gilbreath, S. Binari, and D. Klotzkin, "45-Mbit/s cat's eye modulating retro-reflectors", Opt. Eng. 46 (10), 104001, 2007.

[13] G. C. Gilbreath, W.S. Rabinovich, T.J. Meehan, M.J. Vilcheck, R. Mahon et al., "Large-aperture multiple quantum well modulating retroreflector for free-space optical data transfer on unmanned aerial vehicles", SPIE Opt. Eng.,vol. 40(7), pp. 1348-1356, 2001.

[14] National Academies of Sciences, Engineering, and Medicine, "Achieving Science with CubeSats: Thinking Inside the Box (2016)", Washington, 2016.

[15] Q. Wang, B. Noharet, S. Junique, S. Almqvist, D. Agren and J. Y. Andersson, "1550 $\mathrm{nm}$ transmissive/reflective surface-normal electroabsorption modulator arrays", IEEE Elec. Lett., vol. 42 (1), pp. 47 63, Jan. 2006.

[16] C. Quintana, G. Erry, A. Gomez, Y. Thueux, G. E. Faulkner, and D. C. O’Brien, "Design of a holographic tracking module for long-range retroreflector free-space systems," Appl. Opt., vol. 55, pp. 7173-7178, 2016.

[17] F. Chang, K. Onohara, and T. Mizuochi, "Forward error correction for 100 G transport networks", IEEE Commun. Mag., vol. 48 (3), pp. S48S55, Mar. 2010.

[18] B. Moision, B. I. Erkmen, W. Farr, S. J. Dolinar and K. M. Birnbaum, "Limits on achievable dimensional and photon efficiencies with intensitymodulation and photon-counting due to non-ideal photon-counter behaviour", Proc. SPIE 8246, Free-Space Laser Communication Technologies XXIV, 824609, Feb. 2012. 